\title{
An Improved Path-Generating Regulator for Two-Wheeled Robots to Track the Circle/Arc Passage
}

\author{
Jun Dai, ${ }^{1}$ Naohiko Hanajima, ${ }^{2}$ Toshiharu Kazama, ${ }^{2}$ and Akihiko Takashima ${ }^{3}$ \\ ${ }^{1}$ Division of Production and Information Systems Engineering, Muroran Institute of Technology, 27-1 Mizumoto-cho, Muroran, \\ Hokkaido 050-8585, Japan \\ ${ }^{2}$ College of Design and Manufacturing Technology, Muroran Institute of Technology, 27-1 Mizumoto-cho, Muroran, \\ Hokkaido 050-8585, Japan \\ ${ }^{3}$ Department of Mechanical Engineering, Hokkaido University of Science, 4-1 Maeda 7-jo 15-chome, Teine-ku, \\ Sapporo 006-8585, Japan
}

Correspondence should be addressed to Jun Dai; daijun0922@126.com

Received 9 April 2014; Revised 25 June 2014; Accepted 9 July 2014; Published 23 July 2014

Academic Editor: Anders Eriksson

Copyright (C) 2014 Jun Dai et al. This is an open access article distributed under the Creative Commons Attribution License, which permits unrestricted use, distribution, and reproduction in any medium, provided the original work is properly cited.

\begin{abstract}
The improved path-generating regulator (PGR) is proposed to path track the circle/arc passage for two-wheeled robots. The PGR, which is a control method for robots so as to orient its heading toward the tangential direction of one of the curves belonging to the family of path functions, is applied to navigation problem originally. Driving environments for robots are usually roads, streets, paths, passages, and ridges. These tracks can be seen as they consist of straight lines and arcs. In the case of small interval, arc can be regarded as straight line approximately; therefore we extended the PGR to drive the robot move along circle/arc passage based on the theory that PGR to track the straight passage. In addition, the adjustable look-ahead method is proposed to improve the robot trajectory convergence property to the target circle/arc. The effectiveness is proved through MATLAB simulations on both the comparisons with the PGR and the improved PGR with adjustable look-ahead method. The results of numerical simulations show that the adjustable look-ahead method has better convergence property and stronger capacity of resisting disturbance.
\end{abstract}

\section{Introduction}

Over the last few years, the development on robots has been paid close attention to. There are some research projects such as cleaner robot of iRobot (IRBT) [1], office robot of double robotics [2], and remote-presence robot of mobile access consultation services [3]. These two-wheeled robots have common characteristic that move along the given route. So path tracking serves as an essential task for such autonomous robots.

For circle/arc tracking problem, many approaches have been proposed, a dual estimation algorithm estimated the robot's position and wheel slips based on the Kalman filtering [4], but it is necessary to have previous knowledge about the system and measuring devices. A block iterative method known as four point-explicit group via nine-point Laplacian (4EG9L) was used for solving robot path planning problem [5]. Most such approaches design the feedback control system by converting variables such as input conversion of the mathematical model to a format called chained form. It is noteworthy that the conversion variables cannot be defined globally in such approaches.

Compared with the other papers, the originality of this paper proposed the PGR and improved PGR with adjustable look-ahead method to track the circle/arc for two-wheeled robot. It is a control method that carries out asymptotic convergence of nonholonomic robots to a given path function group.

Two-wheeled robots belong to nonholonomic constraints system [6], which makes it difficult for robots to converge to the target state by deriving a control law [7]. In one of our previous works, we proposed the path-generating regulator (PGR) method, which controls the robot to move forward to the tangential direction of the curve which passes through the robot current position among the family of path functions [8-10]. This method allows us to make the robot 


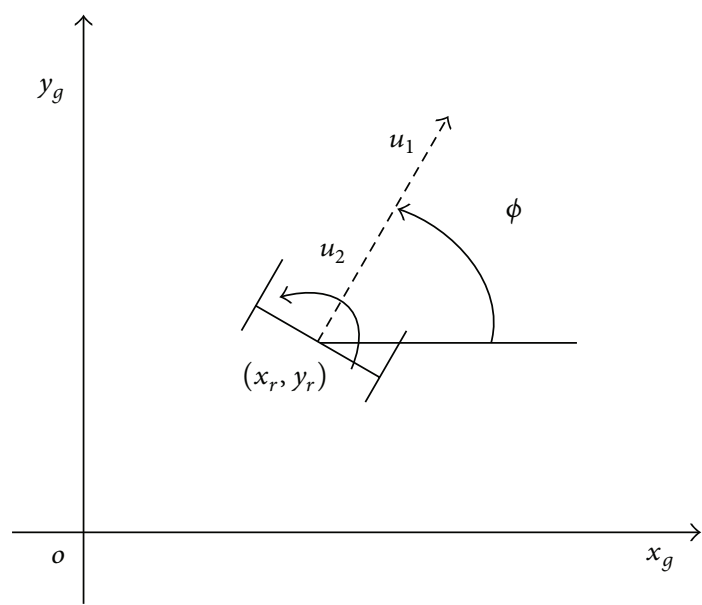

FIgURE 1: Two-wheeled robot mathematic model.

stop at the origin of the rectangular coordinate system. Simultaneously, the global asymptotic stability of PGR has been proven. Because driving environments for robots are usually roads, streets, passages, and ridges. These tracks can be seen as they consist of straight lines and arcs. Recently, the PGR has been extended to path tracking problem along straight passage for two-wheeled robots [11] and the validity has been verified by simulations and experiments. Therefore, we further investigate the PGR and the improved PGR with adjustable look-ahead algorithm to track the circle/arc passage in this paper.

The remainder of this paper is organized as follows. Section 2 reviews the PGR along straight passage for twowheeled robots. In Section 3, we propose the PGR along circle/arc passage based on line approximation in a small interval. Because the robot's trajectory tends to deviate outward from the target circle, the improved PGR along circle/arc passage with adjustable look-ahead method is elaborated in Section 4. In order to verify the efficiency of the two proposed PGR methods, the numerical simulations are executed and discussed in Section 5. Concluding remarks are presented in Section 6.

\section{Review of the PGR along Straight Passage Method for Two-Wheeled Robots}

The PGR was used to solve the path tracking problem along straight passage for two-wheeled robots. To illustrate this method clearly, we establish the robot's mathematic model, definite the path functions, and carry out necessary mathematical calculation and then deduce the steering angle and the moving speed formulas.

2.1. Mathematic Model. In this research, the mathematic model is shown in Figure 1. The kinematic variables of the two- wheeled robot are as follows: $x_{r}$ and $y_{r}$ are the coordinates of the robot in the world reference frame. The translational velocity of the center of the robot $u_{1}$ is related to the velocity in the $x$ and $y$ directions, $\dot{x}_{r}$ and $\dot{y}_{r}$, through (1) and

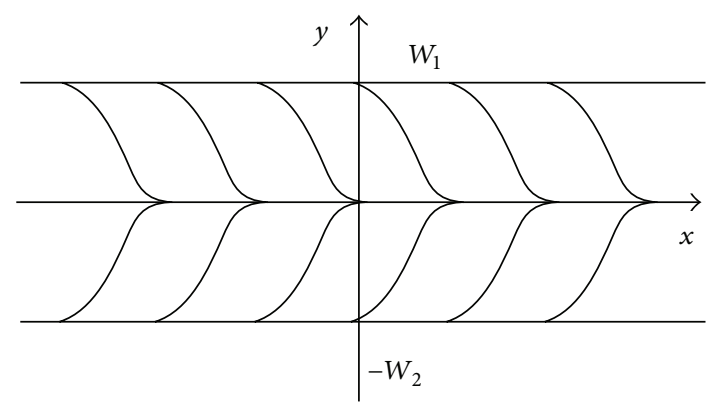

FIGURE 2: The coordinate system and the path functions for straight passage. The robot drives toward the positive direction of the $x$-axis. The horizontal lines $y=W_{1}$ and $y=-W_{2}$ represent the boundary of passage, such as walls.

(2), where $\phi$ is the orientation angle of the robot with respect to the reference frame. The steering angle of the robot $u_{2}$ is the change rate $\dot{\phi}$ of the orientation angle, through (3):

$$
\begin{gathered}
\dot{x}_{r}=u_{1} \cos \phi, \\
\dot{y}_{r}=u_{1} \sin \phi, \\
\dot{\phi}=u_{2} .
\end{gathered}
$$

2.2. Path Functions for Straight Passage. The family of path functions for a half-side of straight passage is defined as the following formula from reference [11]:

$$
y= \begin{cases}W & x<\frac{-\pi+b}{a} \\ \frac{W}{2}(1-\cos (a x-b)) & \frac{\pi+b}{a}<x<\frac{b}{a}, \\ 0 & x>\frac{b}{a},\end{cases}
$$

where $a$ is a positive constant which adjusts the slope of the curve, $b$ is a translation value of path functions family, and $W$ is the half-width of the passage. Instead of $W$, we take $W_{1}$ as the left half width, that is, in the domain of $y>0, W_{2}$ as the right half width, that is, in the domain of $y<0$. When $y$ is negative, the sign of the right side of (4) needs to be changed. The graph of the functions is shown in Figure 2. The robot drives through the passage toward the positive direction of the $x$-axis. $\phi_{r}$ can be expressed as

$$
\phi_{r}= \begin{cases}\tan ^{-1}\left(-a \sqrt{\left(W_{1}-y\right) y}\right) & y \geq 0 \\ \tan ^{-1}\left(a \sqrt{-\left(W_{2}+y\right) y}\right) & y<0 .\end{cases}
$$


Note that $\phi_{r}$ is calculated only by the $y$ coordinate in the region $-W_{2} \leq y \leq W_{1}$. The partial derivative of $\phi_{r}$ with respect to $y$ is calculated as follows:

$$
\frac{\partial \phi_{r}}{\partial y}=\left\{\begin{array}{cl}
-\frac{a\left(W_{1}-2 y\right) \sqrt{\left(W_{1}-y\right) y}}{2 y\left(1+a^{2}\left(W_{1}-y\right) y\right)\left(W_{1}-y\right)} & y \geq 0 \\
\frac{a\left(W_{2}+2 y\right) \sqrt{-\left(W_{2}+y\right) y}}{2 y\left(1-a^{2}\left(W_{2}+y\right) y\right)\left(W_{2}+y\right)} & y<0 .
\end{array}\right.
$$

2.3. Steering Angle $u_{2}$. The deviation between the target angle $\phi_{r}$ and the actual angle $\phi$ is set as $\delta$ :

$$
\delta=\phi-\phi_{r} .
$$

Under the control of $u_{2}, \delta$ obeys the following derivative equation of the first order delay system:

$$
\dot{\delta}=-\lambda \delta,
$$

where $\lambda$ is a coefficient constant, when $\delta$ converges to $0 ; \phi$ approaches $\phi_{r}$ simultaneously. According to (3), (6), and (8), we obtain $u_{2}$ for straight passage as follows:

$$
\begin{aligned}
u_{2}= & \begin{cases}g_{1}(y, \phi) & 0<y<W_{1}-\varepsilon \\
g_{2}(y, \phi) & -W_{2}+\varepsilon<y<0,\end{cases} \\
g_{1}(y, \phi)= & -\lambda\left(\phi+\tan ^{-1}\left(a \sqrt{\left(W_{1}-y\right) y}\right)\right) \\
& -\frac{a\left(W_{1}-2 y\right) \sqrt{\left(W_{1}-y\right) y}}{2 y\left(1+a^{2}\left(W_{1}-y\right) y\right)\left(W_{1}-y\right)} u_{1} \sin \phi, \\
g_{2}(y, \phi)= & -\lambda\left(\phi-\tan ^{-1}\left(a \sqrt{-\left(W_{2}+y\right) y}\right)\right) \\
& +\frac{a\left(W_{2}+2 y\right) \sqrt{-\left(W_{2}+y\right) y}}{2 y\left(1-a^{2}\left(W_{2}+y\right) y\right)\left(W_{2}+y\right)} u_{1} \sin \phi,
\end{aligned}
$$

where $\varepsilon$ is a small positive constant. To avoid division by zero in computer calculation of (10) and (11), when the value of $y$ is around $0, W_{1}$ or $W_{2}$ and the speed command $u_{1}$ need to be nonzero value.

2.4. Translational Velocity $u_{1} \cdot u_{1}$ is derived by Lyapunov's stability method. A hybrid continuous control algorithm in (12), of which the second part puts more emphasis on advance close to the $x$-axis and the first part guarantees stability in other location, is applied. The control algorithm can be expressed as follows:

$$
u_{1}=-\left(1-K_{m} e^{-c_{m} y^{2}}\right) \frac{1-e^{-c y \sin \phi}}{1+e^{-c y \sin \phi}} V_{m}+K_{m} e^{-c_{m} y^{2}} V_{m},
$$

where $K_{m} e^{-c_{m} y^{2}}$ is the modification coefficient used to adjust the emphasis between two parts. $K_{m}$ is within the limit of $0 \leq K_{m} \leq 1$ and $e^{-c_{m} y^{2}}$ will be equal to 1 on the $x$-axis and close to 0 away from the $x$-axis. $c_{m}$ is an adjustable parameter and $c_{m}>0$.

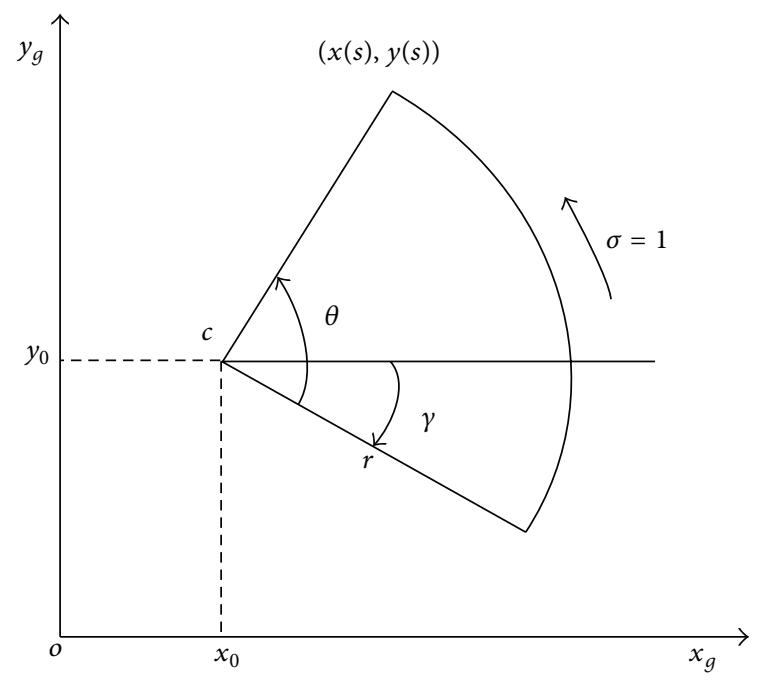

FIgURE 3: The path of circle/arc.

\section{The PGR along Circle/Arc Passage Method}

In a small interval, circle/arc can be seen as line approximately. The PGR along circle/arc passage based on the theory of straight passage is proposed in this section.

As shown in Figure 3, we set the circle/arc path functions as

$$
\begin{aligned}
& x(s)=x_{0}+r \cos \left(\sigma \frac{s}{r}+\gamma\right), \\
& y(s)=y_{0}+r \sin \left(\sigma \frac{s}{r}+\gamma\right),
\end{aligned}
$$

where $s$ is the length of circle/arc, $r$ is the radius of circle/arc, and $\gamma$ is the inclination angle of circle/arc. $c\left(x_{0}, y_{0}\right)$ is the center of circle/arc. $\sigma$ determines the move direction of circle/arc, $\sigma=1$ means counterclockwise direction, and $\sigma=$ -1 means clockwise direction. $\theta$ is central angle, $0<\theta \leq 2 \pi$, and $\theta=s / r$.

The global and local coordinate systems are shown in Figure 4. $o-x_{g} y_{g}$ is the global coordinate system and $p_{l}$ $x_{l} y_{l}$ is the local coordinate system. $\left(x_{r}, y_{r}, \phi\right)$ is the pose of robot in the global coordinate system. $c\left(x_{0}, y_{0}\right)$ is the central point of circle/arc. The angle between the line which passes through $\left(x_{r}, y_{r}\right)$ and $c\left(x_{0}, y_{0}\right)$ and $x$-axis is named as $\alpha$ that is expressed as (14). The width of passage inside of the circle/arc is $W_{1}$ and $W_{1}<r$; the opposite side is $W_{2}$ :

$$
\alpha=\tan ^{-1}\left(\frac{y_{r}-y_{0}}{x_{r}-x_{0}}\right) .
$$

The theory of PGR along straight passage can be used for circle/arc passage in a small interval $d_{t}$ in the local coordinate system $p_{l}-x_{l} y_{l}$. In order to simplify computation, we select the intersection point $p_{l}\left(x_{l}, y_{l}\right)$, expressed as (15) and (16) and the line through $c\left(x_{0}, y_{0}\right)$ and $\left(x_{r}, y_{r}\right)$ with the circle/arc as the origin of the local coordinate system. $x_{1}$-axis is the tangential direction of the arc and $y_{l}$-axis is the normal 


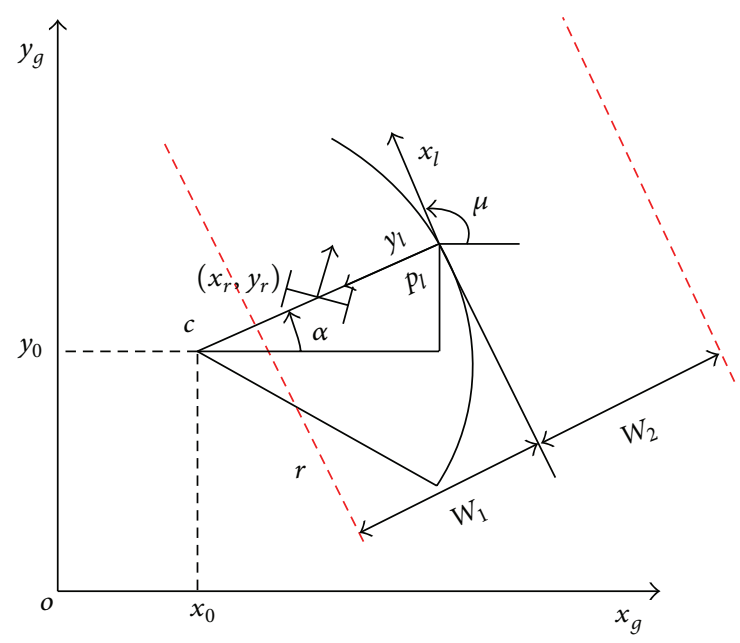

FIgURe 4: Global and local coordinate systems.

direction. $\mu$ means the rotation angle that rotates from the local coordinate system to global coordinate system from (17):

$$
\begin{gathered}
x_{l}=x_{0}+r \cos \alpha, \\
y_{l}=y_{0}+r \sin \alpha, \\
\mu=\frac{\pi}{2}+\alpha .
\end{gathered}
$$

Therefore, we can obtain the pose of robot $\left(x_{r}^{\prime}, y_{r}^{\prime}, \phi^{\prime}\right)$ in the local coordinate system:

$$
\begin{gathered}
x_{r}^{\prime}=x_{r} \cos \mu+y_{r} \sin \mu-x_{l} \cos \mu-y_{l} \sin \mu, \\
y_{r}^{\prime}=-x_{r} \sin \mu+y_{r} \cos \mu+x_{l} \sin \mu-y_{l} \cos \mu, \\
\phi^{\prime}=\phi-\mu .
\end{gathered}
$$

The flow chart is shown in Figure 5. We establish global coordinate system $o-x_{g} y_{g}$ and local coordinate system $p_{l}-x_{l} y_{l}$ according to (14)-(17) firstly. Then the pose of robot $\left(x_{r}, y_{r}, \phi\right)$ is converted to the local coordinate value $\left(x_{r}^{\prime}, y_{r}^{\prime}, \phi^{\prime}\right)$ according to (18). Within the time interval $d_{t}$, the PGR along $x$-axis of local coordinate system is to control robot and a new pose is obtained according to (9) and (12). Then the new pose of robot is converted to global coordinate value according to the inverse transformation of (18). Finally, the robot judges whether the new location is the goal or not. If the new location is not the goal, the new local coordinate system is needed to be established based on the new robot pose and the center of circle/arc. Program is running along this cycle process until the robot reaches the goal.

\section{The Improved PGR with the Adjustable Look-Ahead Method along Circle/Arc Passage}

The PGR is extended to drive the robot move along circle/arc passage based on the straight passage theory in Section 3.

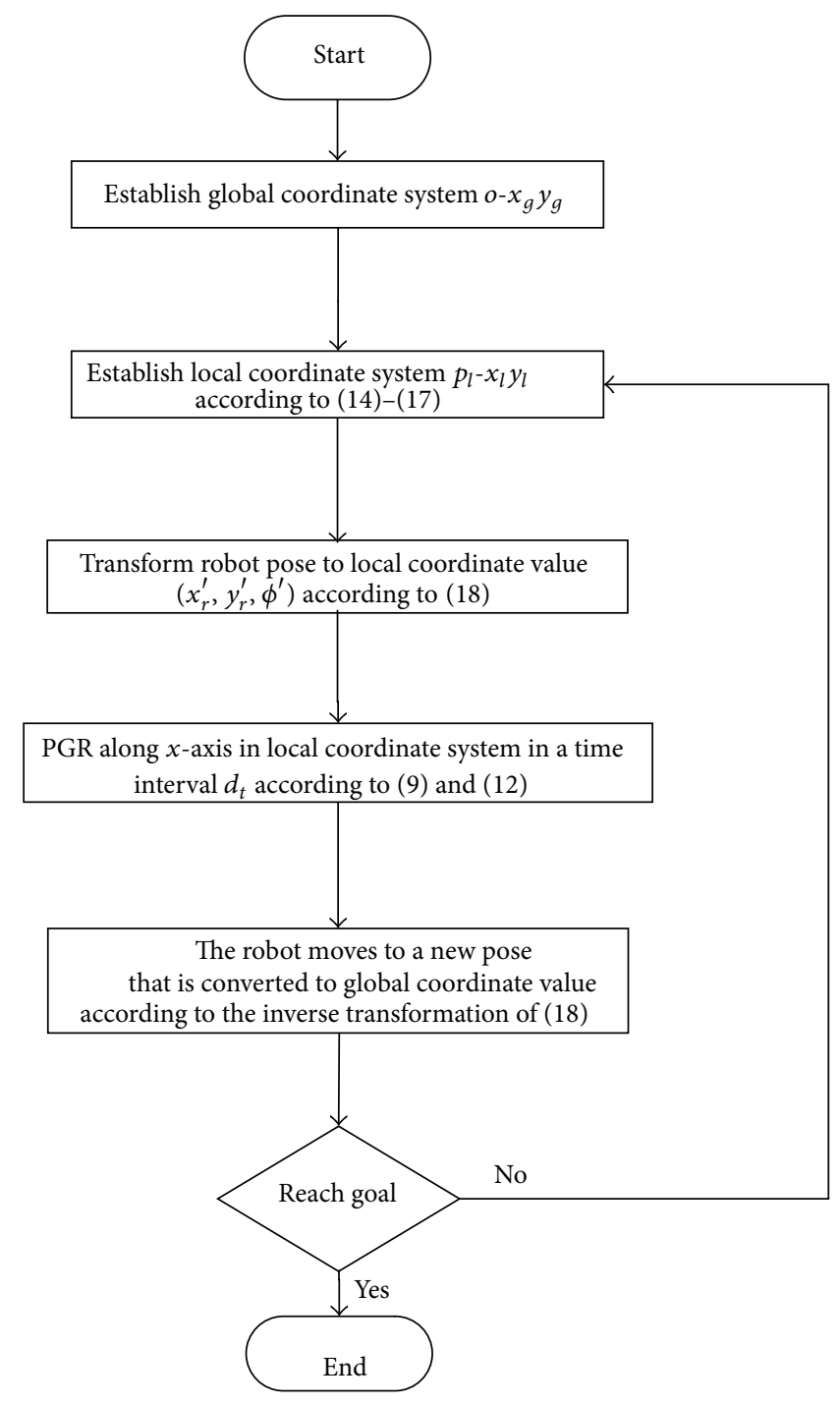

FIgURE 5: Flow chart under PGR along circle/arc passage.

The improved PGR with the adjustable look-ahead method is proposed to make the robot's trajectory converge to the target circle/arc.

Global and local coordinate systems with the adjustable look-ahead method are shown in Figure 6. The distance between the robot location $\left(x_{r}, y_{r}\right)$ and the center of the circle/arc $c\left(x_{0}, y_{0}\right)$ is assumed as $d$ in (19). The central angle between robot location and local coordinate origin is assumed as $\beta$ in (20), which is named as adjustable lookahead central angle, because $\beta$ will change if $d$ changes:

$$
\begin{gathered}
d=\sqrt{\left(x_{r}-x_{0}\right)^{2}+\left(y_{r}-y_{0}\right)^{2}}, \\
\beta=\cos ^{-1}\left(\frac{r}{d}\right) .
\end{gathered}
$$

The origin $p_{l}^{\prime}\left(x_{l}^{\prime}, y_{l}^{\prime}\right)$ which is described in (21) in the new local coordinate system is the tangent point of the circle/arc at the front of the robot location, whose tangent line passes 


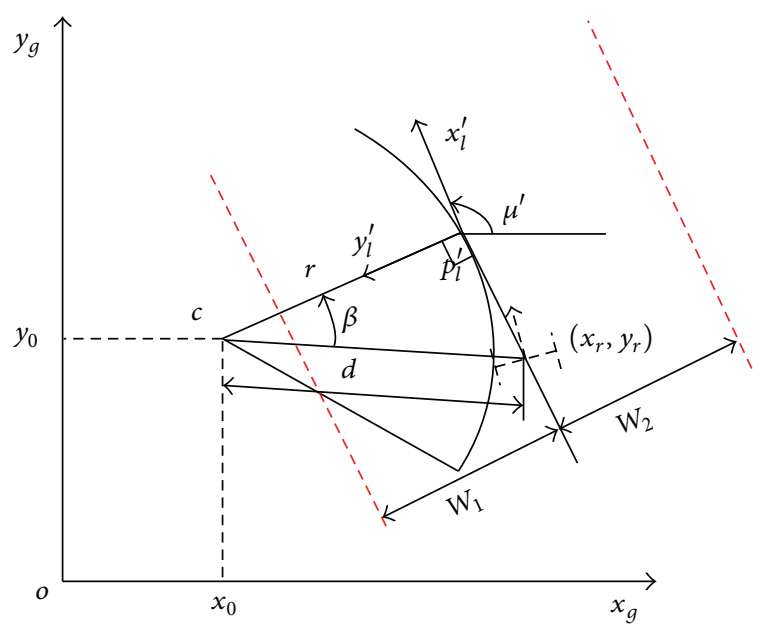

FIgURE 6: Global and local coordinate systems for the improved PGR with the adjustable look-ahead method.

through the $\left(x_{r}, y_{r}\right), x_{l}^{\prime}$-axis is the tangential direction of the arc and $y_{l}^{\prime}$-axis is the normal direction. $\mu^{\prime}$ means the rotation angle that rotates from the new local coordinate system to the global coordinate system:

$$
\begin{gathered}
x_{l}^{\prime}=x_{r}+d \sin \beta \cos \left(\frac{3 \pi}{2}-\beta-\alpha\right), \\
y_{l}^{\prime}=y_{r}+d \sin \beta \sin \left(\frac{3 \pi}{2}-\beta-\alpha\right), \\
\mu^{\prime}=\frac{\pi}{2}+\alpha+\beta .
\end{gathered}
$$

The local coordinate system is established at the front of robot location and is adjusted with the change of $\beta$, so this method is named as adjustable look-ahead method.

The flow chart under the improved PGR with the adjustable look-ahead method along circle/arc passage is shown in Figure 7. The difference with the method in Section 3 is the introduction of $\beta$. When the robot's new location is outside of target circle/arc, that is, $d>r$, the program calculates $\beta$ then the new local coordinate system is established based on (21). Otherwise, when the robot's new location is inside of target circle/arc, that is, $d<r$, the process is the same with the flow chart in Figure 6.

\section{Numerical Simulations}

To confirm the efficiency of the proposed PGR method in Sections 3 and 4 for the robot's path tracking along circle/arc passage, the numerical simulations are performed in several aspects, respectively, in this section.

We investigate the influence of coefficient constant $\lambda$ and the time interval $d_{t}$ to the robot's trajectory. In addition, limited random disturbances are imposed to the inputs of velocity $u_{1}$ and steering angle $u_{2}$ to investigate the robustness of the two methods. The overall simulation results are discussed finally. In the simulation, the simulation time $t$ is set separately as 40 seconds in circle/arc passage and 50 seconds in

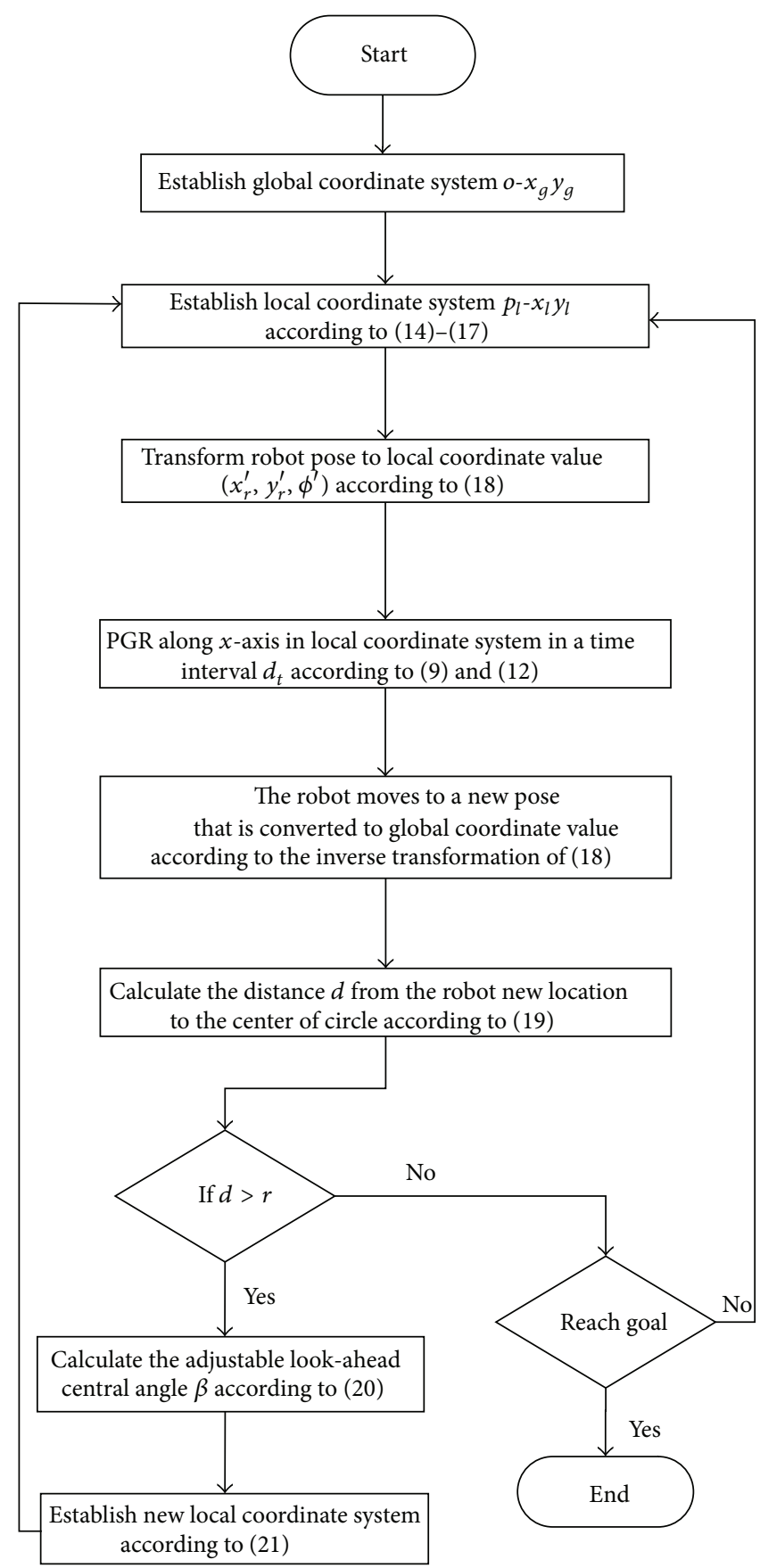

FIGURe 7: Flow chart under the improved PGR with the adjustable look-ahead method along circle/arc passage.

the S-shaped passage; that is to say, when the simulation time $t$ is equal to 40 seconds or 50 seconds, the robot receives a command of velocity $u_{1}=0$ and steering angle $u_{2}=0$ and stops.

The numerical simulations are performed with the software MATLAB 7.11.0 (The MathWorks, Inc.). The default parameter values for the simulations are listed in Table 1 . In the simulation results, the red dashed line of (C) or (D) represents the target circle/arc. The red cross represents the center of circle/arc. The robot's trajectories are shown in the 


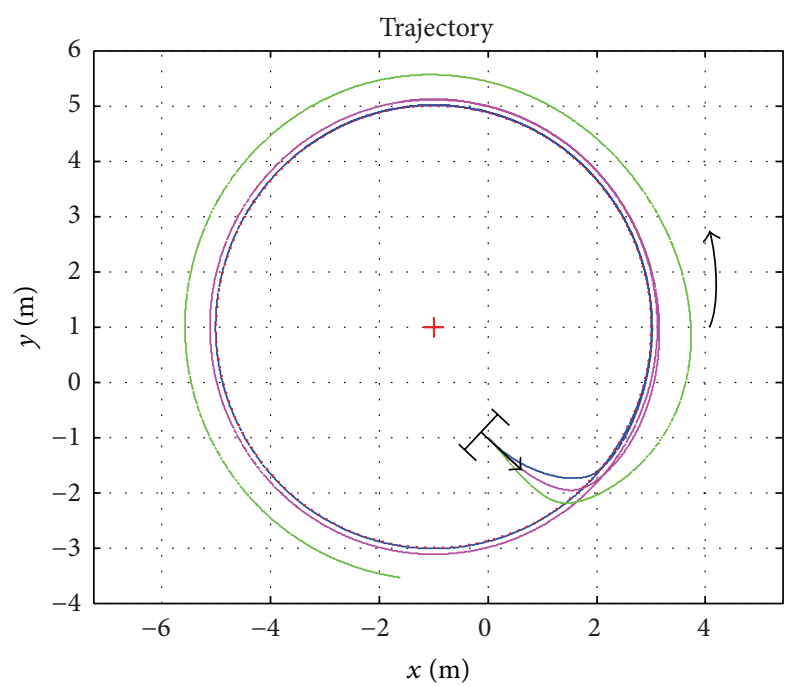

(A)

(B)

(C)

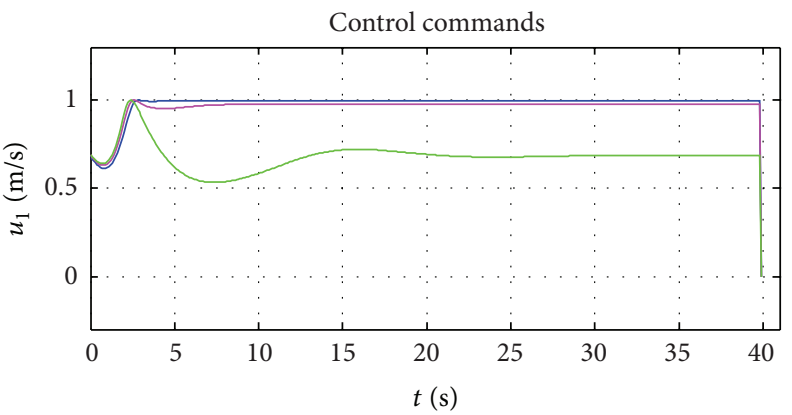

- (A)

(B)

$-(\mathrm{C})$

(a)

(b)

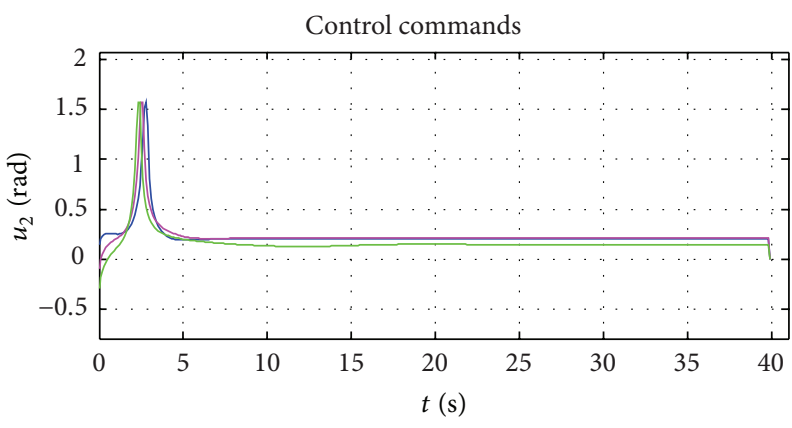

(A)

(c)

Figure 8: Trajectories in the $x-y$ plain and time response of control commands $u_{1}$ and $u_{2}$ by the method in Section 3 at different values of $\lambda$, from $(\mathrm{A})$ to $(\mathrm{C})$.

$x-y$ plain. Initial condition is designated by an icon with an arrow. The thick arrow represents the direction of the robot's motion.

5.1. The Influence of Coefficient Constant $\lambda$. In the numerical simulation process, we only change the value of coefficient constant $\lambda$ and keep the other parameters constant. The parameters are listed in Table 2.

The robot's trajectories and control commands of PGR proposed in Section 3 at different $\lambda$ values are depicted in Figure 8. The value of $\lambda$ is 0.2 for (A), 0.5 for (B), and 0.9 for (C). As described in (8), $\lambda$ is a coefficient constant for adjusting the response speed of $u_{2}$; therefore, the trajecotry tends to converge the target circle if $\lambda$ becomes large. It is found from Figure 8(a) that the convergence property is the best when $\lambda$ is 0.9 , but the trajectory does not yet converge to the target circle/arc.
TABLE 1: Default parameter values for numerical simulations.

\begin{tabular}{lcc}
\hline Parameters & Values & Unit \\
\hline$r$ & 4 & $\mathrm{~m}$ \\
$W_{1}, W_{2}$ & 2.5 & $\mathrm{~m}$ \\
$a$ & 1 & - \\
$\varepsilon$ & 0.001 & - \\
$V_{m}$ & 1 & $\mathrm{~m} / \mathrm{s}$ \\
$c$ & 1 & - \\
$c_{m}$ & 1 & - \\
$k_{m}$ & 1 & - \\
$\gamma$ & $\pi / 4$ & $\mathrm{rad}$ \\
$\theta$ & $2 \pi$ & $\mathrm{rad}$ \\
Initial condition & $(0,-1,-\pi / 4)$ & - \\
\hline
\end{tabular}



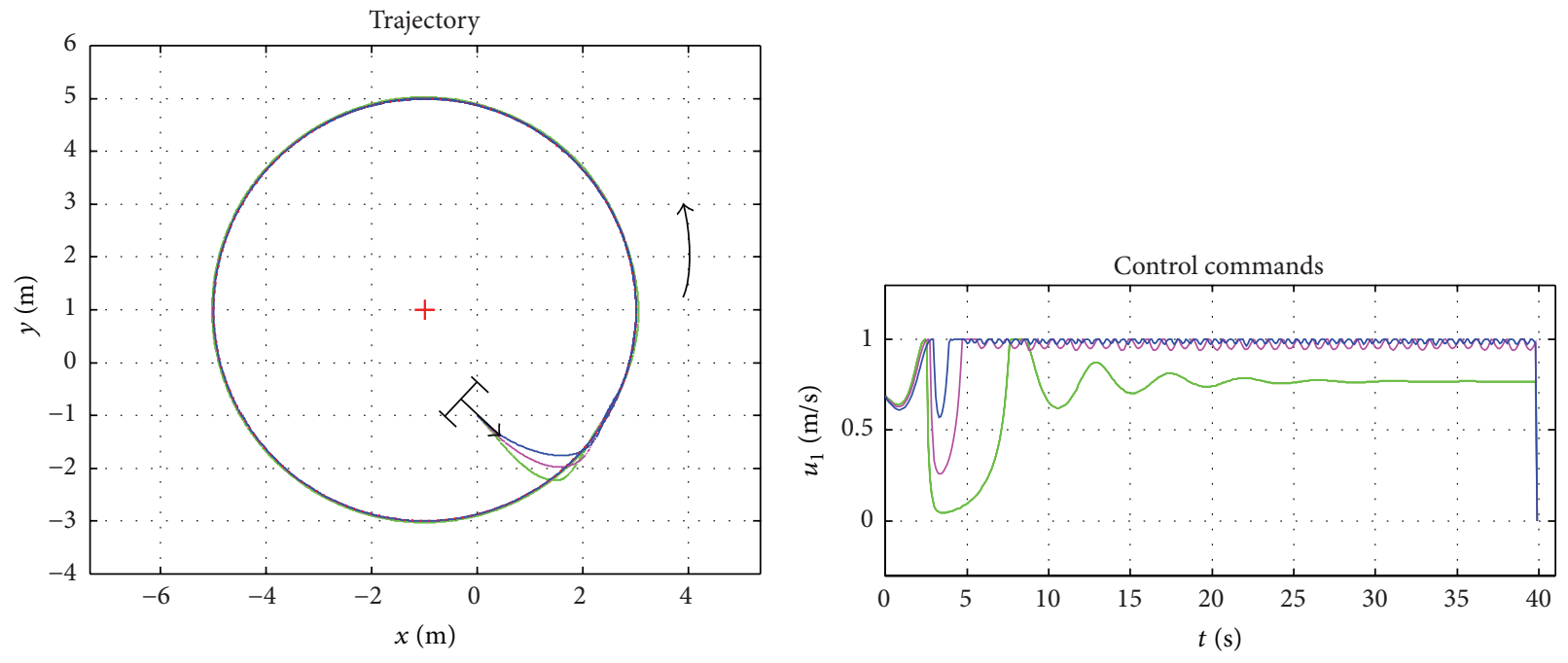

(A)

(C)

- (A)

(B)

(D)

- (B)

$-(\mathrm{C})$

(a)

(b)

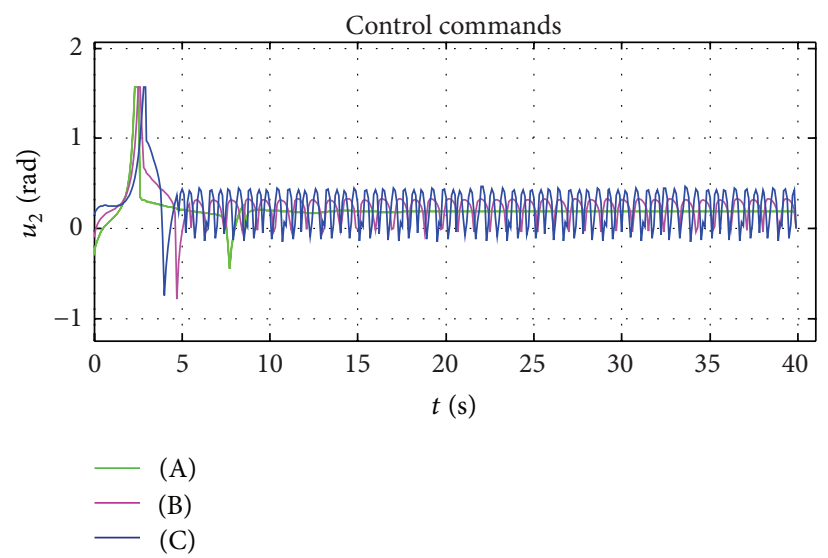

(c)

FIGURE 9: Trajectories in the $x$ - $y$ plain and time response of control commands $u_{1}$ and $u_{2}$ by the PGR with the adjustable look-ahead method at different values of $\lambda$, from $(\mathrm{A})$ to $(\mathrm{C})$.

TABLE 2: Default parameter values for numerical simulations.

\begin{tabular}{ccccc}
\hline No. & $\lambda$ & $d_{t}$ & $t$ & Color \\
\hline (A) & 0.2 & $0.1 \mathrm{~s}$ & $40 \mathrm{~s}$ & Green \\
(B) & 0.5 & $0.1 \mathrm{~s}$ & $40 \mathrm{~s}$ & Magenta \\
(C) & 0.9 & $0.1 \mathrm{~s}$ & $40 \mathrm{~s}$ & Blue \\
\hline
\end{tabular}

Figure 9 depicts the robot's trajectories and control commands of the improved PGR with the adjustable look-ahead method at different $\lambda$ values. The value of $\lambda$ is 0.2 for (A), 0.5 for (B), and 0.9 for (C). It is observed from Figure 9(a) that even if $\lambda$ is large or small, the trajectories can converge to the target circle/arc well, which indicates that the value of $\lambda$ has no influence to the convergence property. The improved PGR with the adjustable look-ahead method has improved the performance of path tracking along circle/arc passage for robot. In Figures 9(b) and 9(c), the values of $u_{1}$ and $u_{2}$ have small fluctuation after 5 seconds when $\lambda$ is 0.5 and 0.9 , respectively. The fluctuation range of $u_{1}$ is $0.04 \mathrm{~m} / \mathrm{s}$ when $\lambda$ is 0.5 and $0.09 \mathrm{~m} / \mathrm{s}$ when $\lambda$ is 0.9 . Because the fluctuation range is very little, it can be ignored. The fluctuation range of $u_{2}$ is $0.3 \mathrm{rad}$ when $\lambda$ is 0.5 and $0.5 \mathrm{rad}$ when $\lambda$ is 0.9 . It is considered that the fluctuation is caused by the local coordinate system changing constantly and fluctuation range of $u_{2}$ has proportional relation with $\lambda$.

5.2. The Influence of Time Intervals $d_{t}$. In this numerical simulation, three values of time interval $d_{t}$ are given. The parameters are listed in Table 3.

The robot's trajectories and control commands of PGR proposed in Section 3 at different time intervals $d_{t}$ are 


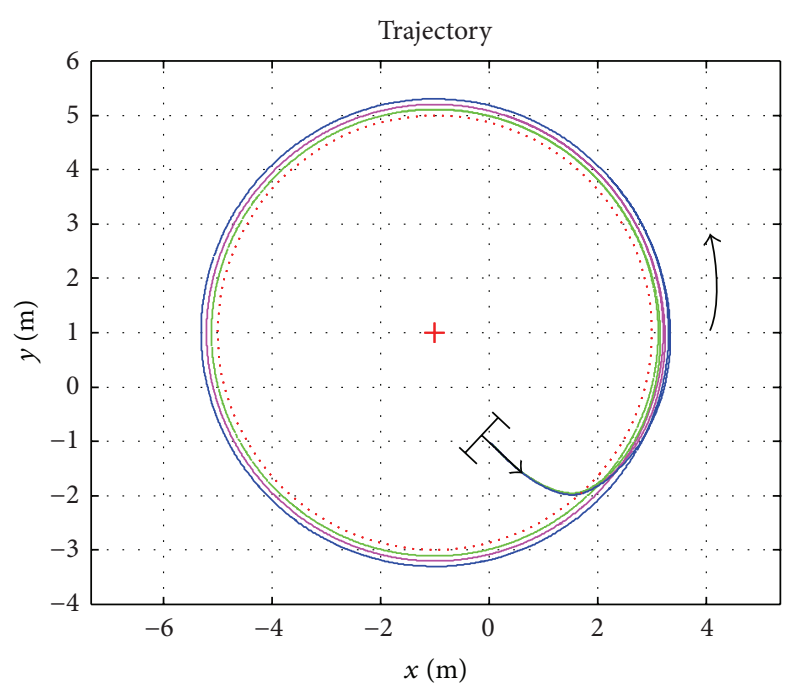

(A)

(B)
(C)

(D)

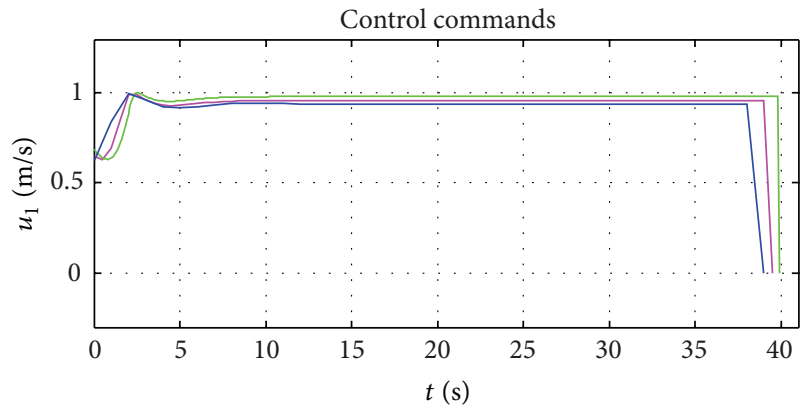

(A)

(B)

(a)

(b)

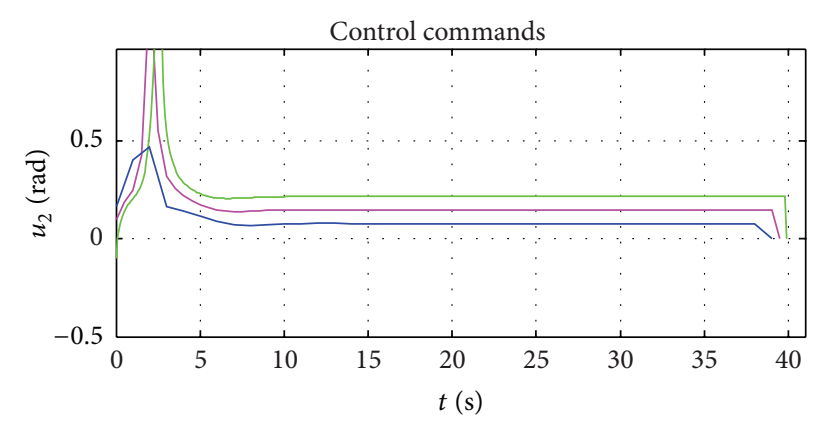

(A)

(B)

(C)

(c)

FIGURE 10: Trajectories in the $x-y$ plain and time response of control commands $u_{1}$ and $u_{2}$ by the method in Section 3 at different time interval values of $d_{t}$, from (A) to (C).

TABLE 3: Default parameter values for numerical simulations.

\begin{tabular}{ccccc}
\hline No. & $d_{t}$ & $\lambda$ & $t$ & Color \\
\hline (A) & $0.1 \mathrm{~s}$ & 0.5 & $40 \mathrm{~s}$ & Green \\
(B) & $0.5 \mathrm{~s}$ & 0.5 & $40 \mathrm{~s}$ & Magenta \\
(C) & $1 \mathrm{~s}$ & 0.5 & $40 \mathrm{~s}$ & Blue \\
\hline
\end{tabular}

depicted in Figure 10. The value of $d_{t}$ is $0.1 \mathrm{~s}$ for $(\mathrm{A}), 0.5 \mathrm{~s}$ for (B), and $1 \mathrm{~s}$ for $(\mathrm{C})$. When the value of time interval $d_{t}$ becomes short, the move distance of the robot becomes short and the orientation is unchanged. The results point out that the convergence property tends to be better when the time interval $d_{t}$ becomes shorter, but the trajectory is not able to converge to the target circle/arc. The velocity $u_{1}$ tends to be stable after 8 seconds. The steering angle $u_{2}$ tends to be stable after 6 seconds.

Figure 11 depicts the robot's trajectories and control commands by improved PGR with the adjustable look-ahead method at different $d_{t}$ values. The value of $d_{t}$ is $0.1 \mathrm{~s}$ for (A), $0.5 \mathrm{~s}$ for (B), and $1 \mathrm{~s}$ for (C). From Figure 11(a), we discover when the value of $d_{t}$ is $1 \mathrm{~s}$ the trajectory in the interval of $d_{t}$ tends to become straight line and the trajectory fluctuates around the target circle/arc, which indicates that the convergence property becomes poor when $d_{t}$ becomes long. If we chose the proper $d_{t}$ value, the robot trajectory is able to converge to the target circle/arc perfectly. Figures 11(b) and 11 (c) show that the fluctuation range of velocitiy $u_{1}$ tends to be wide when the value of $d_{t}$ becomes long. The steering angles $u_{2}$ have the same fluctuation range with the different values of $d_{t}$, but the fluctuation period tends to be long when $d_{t}$ becomes long. 


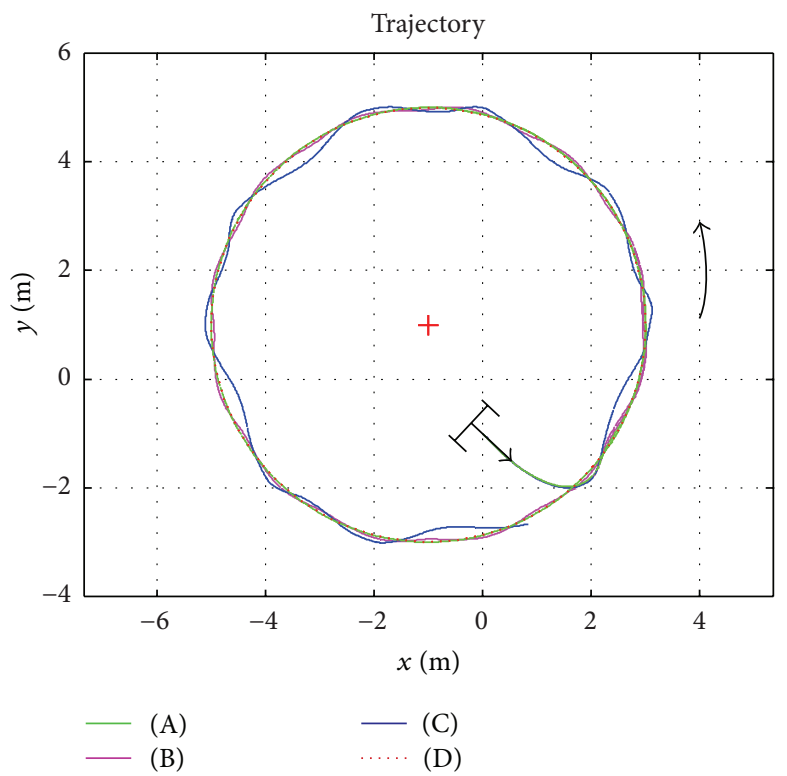

(a)

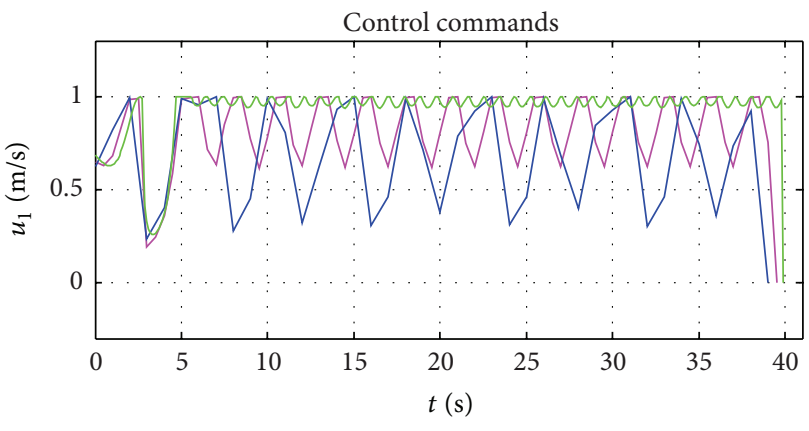

(A)

(B)

(b)

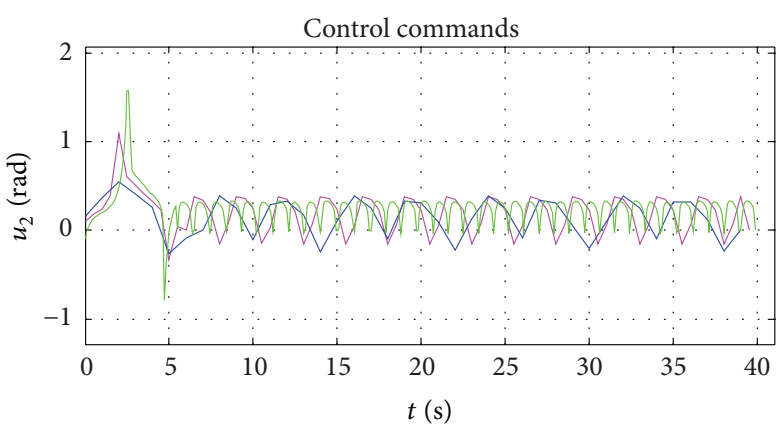

(A)

(B)

(C)

(c)

FIGURE 11: Trajectories in the $x-y$ plain and time response of control commands $u_{1}$ and $u_{2}$ by improved PGR with the adjustable look-ahead method at different time interval values of $d_{t}$, from (A) to (C).

5.3. The Influence with Disturbance Imposed on the Inputs of $u_{1}$ and $u_{2}$. In fact, because of the restrictions of robot itself and external environment, the inputs are often accompanied by some disturbance. For instance, when the robot runs on uneven ground, the steering wheel may receive disturbance force from the ground. To investigate the robustness of the two proposed PGR methods, two different sizes limited random disturbances $e_{1}$ and $e_{2}$ (listed in Table 4) are imposed to the inputs of velocity command $u_{1}$ and steering angle command $u_{2}$, respectively.

The robot's trajectories and control commands by the method proposed in Section 3 at different disturbances are depicted in Figure 12. The trajectories are the same with the two different disturbances, which indicates that this method has strong capacity of resisting disturbance. The fluctuation range of control commands tends to be wide with the increasement of disturbance.
TABLE 4: Default parameter values for numerical simulations.

\begin{tabular}{cccccc}
\hline No. & $e_{1}, e_{2}$ & $\lambda$ & $d_{t}$ & $t$ & Color \\
\hline (A) & $0.2 \times \operatorname{rand}(1)-0.1$ & 0.5 & $0.1 \mathrm{~s}$ & $40 \mathrm{~s}$ & Blue \\
(B) & $0.5 \times \operatorname{rand}(1)-0.1$ & 0.5 & $0.1 \mathrm{~s}$ & $40 \mathrm{~s}$ & Green \\
\hline
\end{tabular}

Similarly, Figure 13 depicts the robot's trajectories and control commands by the improved PGR with the adjustable look-ahead method at different disturbances. The results point out that the trajectories converge to the target perfectly with the two different disturbances, which indicates that the improved PGR has strong capacity of resisting disturbance as well. The fluctuation range of control commands tends to be wide with the increasement of disturbance. 


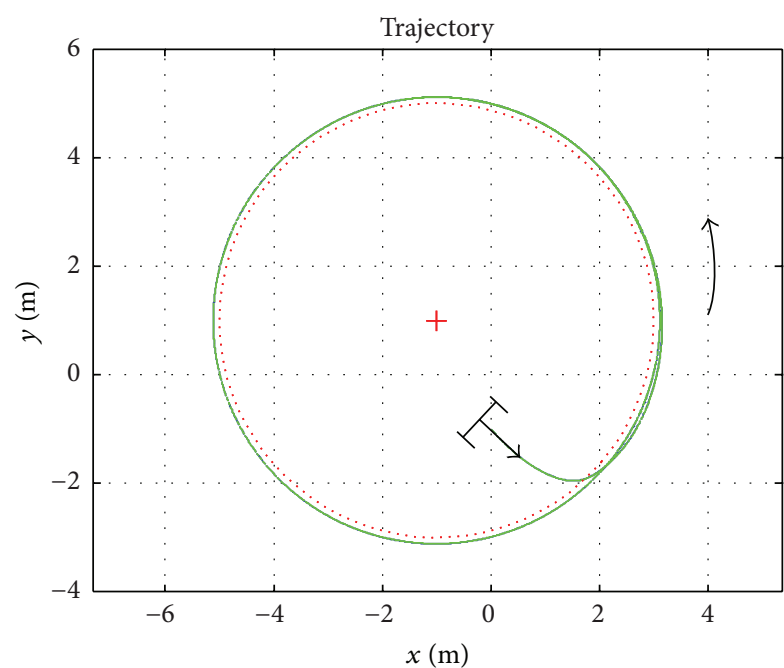

(A)

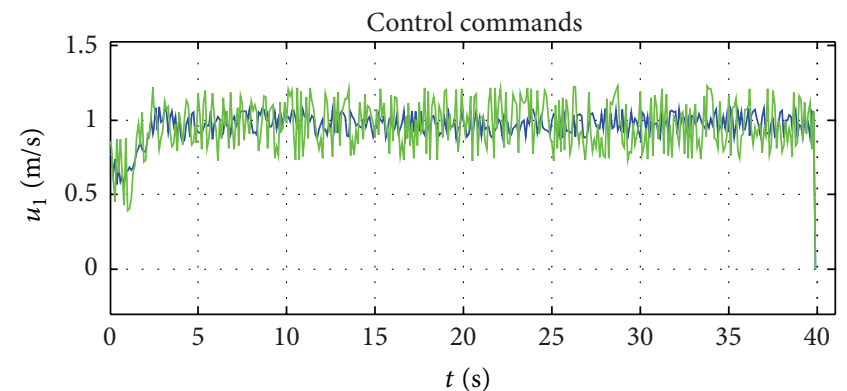

(A)

(B)

(a)

(b)

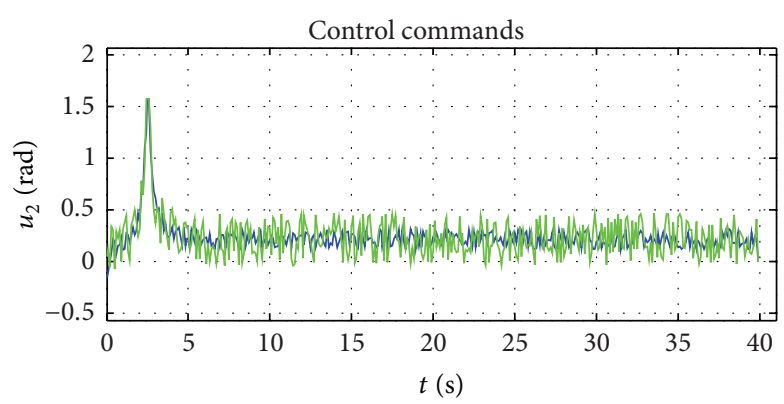

(A)

(B)

(c)

FIGURE 12: Trajectories in the $x-y$ plain and time response of control commands of PGR proposed in Section 3 at different disturbance values of $e_{1}$ and $e_{2}$, from (A) to (B).

5.4. The PGR and Improved PGR with Adjustable Look-Ahead Method to Track the S-Shaped Passage. In this section we apply that the PGR and the improved PGR with the adjustable look-ahead method applied to track the S-shaped passage.

Shown in Figure 14, the S-shaped passage consists of two arcs. The centers of two arcs are $o_{1}\left(x_{1}, y_{1}\right)$ and $o_{2}\left(x_{2}, y_{2}\right)$, the radiuses are $r_{1}$ and $r_{2}$, and the intersection point of two arcs is $p\left(x_{p}, y_{p}\right)$ that can be solved by (22) and (23):

$$
\begin{aligned}
& x_{p}=\frac{1}{2}\left(x_{1}+x_{2}\right), \\
& y_{p}=\frac{1}{2}\left(y_{1}+y_{2}\right) .
\end{aligned}
$$

Firstly, the robot moves along the arc $o_{1}$ counterclockwise, when it reaches the intersection point $p$; then it starts from $p$ and moves along the $\operatorname{arc} \mathrm{O}_{2}$ clockwise.

The condition (A) does not consider error in input and the condition (B) imposes the disturbance on the inputs
TABLE 5: Default parameter values for numerical simulations.

\begin{tabular}{lccccc}
\hline No. & Method & $\lambda$ & $d_{t}$ & $t$ & Color \\
\hline (A) & The PGR & 0.35 & $0.1 \mathrm{~s}$ & $50 \mathrm{~s}$ & blue \\
(B) & $\begin{array}{c}\text { The improved } \\
\text { PGR }\end{array}$ & 0.35 & $0.1 \mathrm{~s}$ & $50 \mathrm{~s}$ & green \\
\hline
\end{tabular}

of $u_{1}$ and $u_{2}$. The default parameter values for numerical simulations are shown in Table 5.

Figure 15 depicts the robot's trajectories and control commands by the PGR and the improved PGR with adjustable look-ahead method to track the circle/arc passage. As can be seen in the upper graph, the trajectory by the PGR is distributed in the outside of the target S-shaped passage and the trajectories converge to the target perfectly by the improved PGR with adjustable look-ahead method. In the below graph, around the intersection point $p$, the steering angle has two sudden changes from 29 seconds to 31 seconds. 


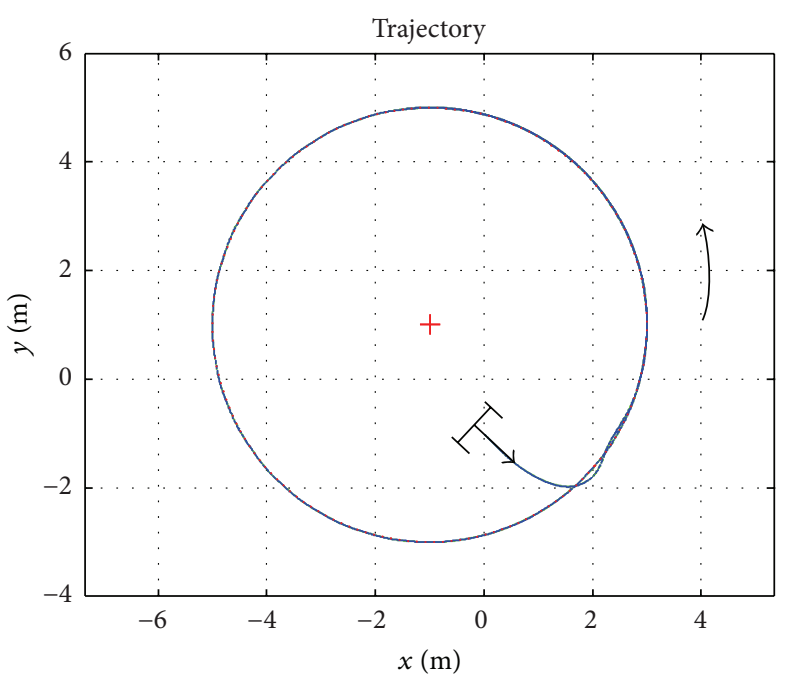

- (A)

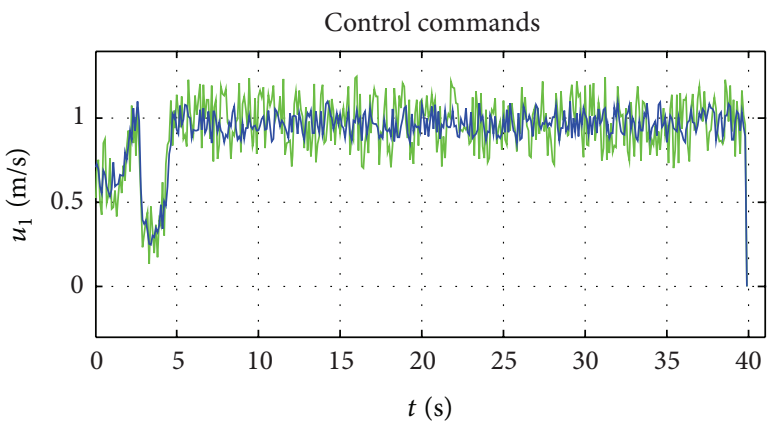

(A)

(B)

(a)

(b)

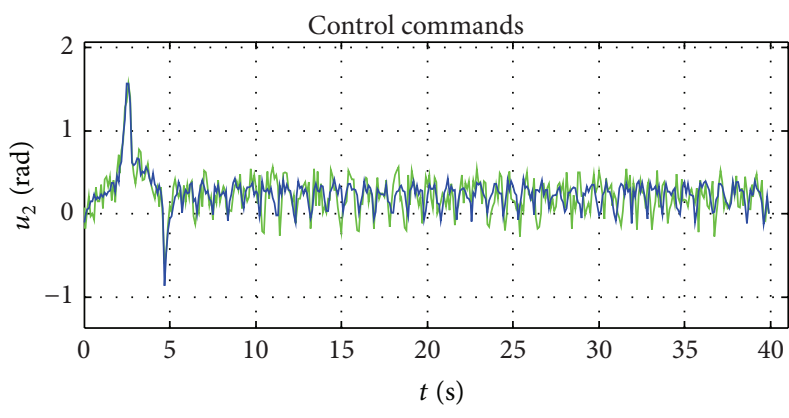

- (A)

- (B)

(c)

FIGURE 13: Trajectories in the $x-y$ plain and time responses of control commands by improved PGR with the adjustable look-ahead method for robot at different disturbance values of $e_{1}$ and $e_{2}$, from (A) to (B).

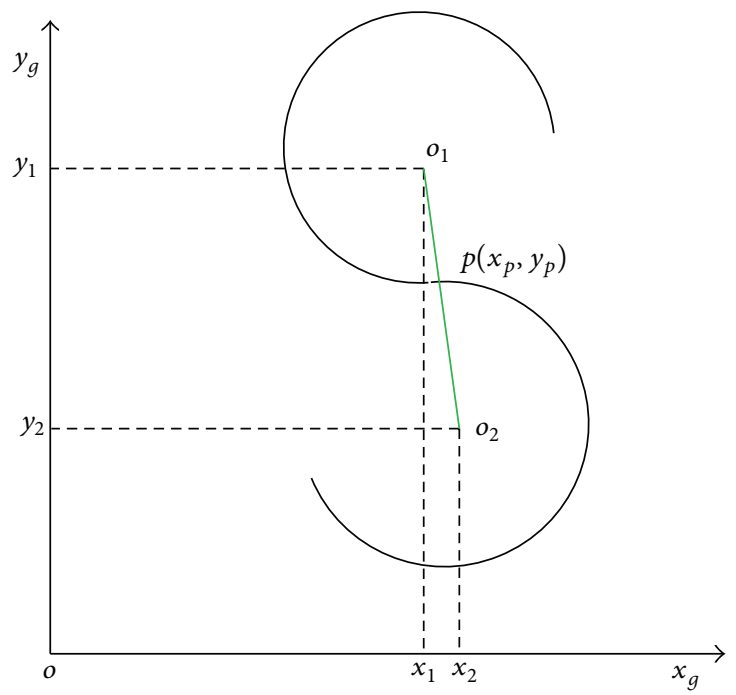

Figure 14: The S-shaped model. 


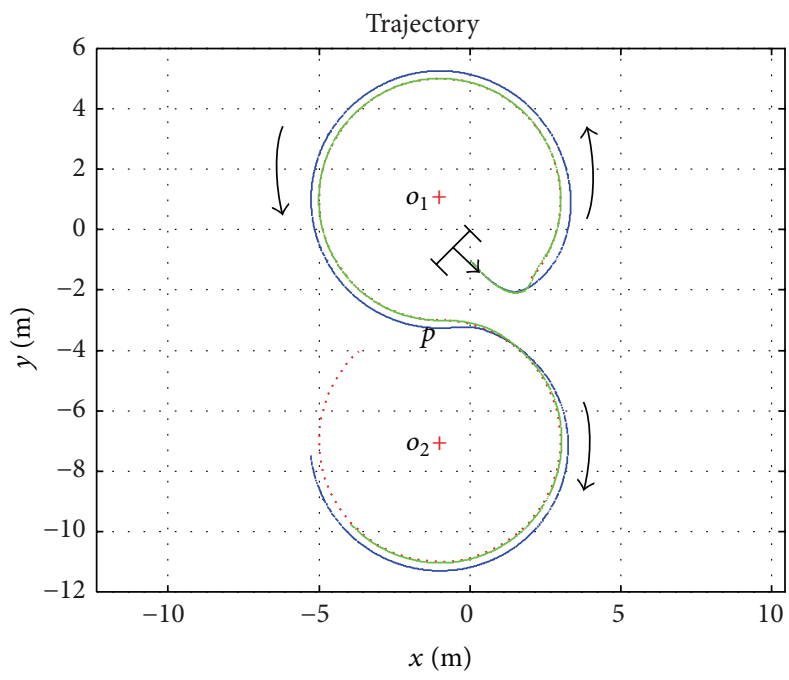

(A)

(C)

(a)

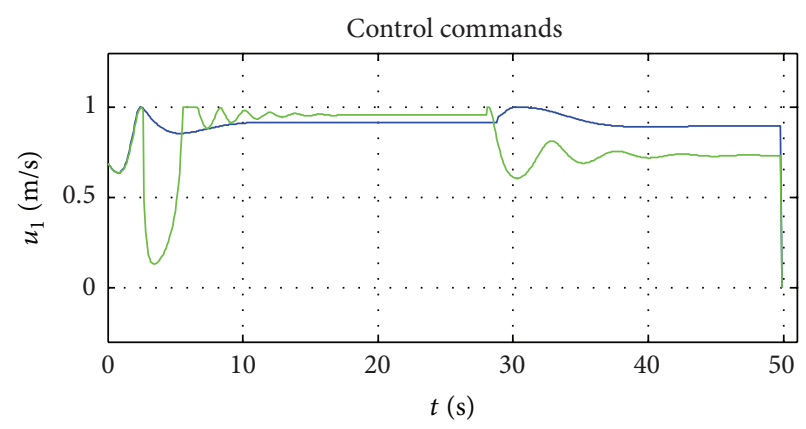

(A)

(B)

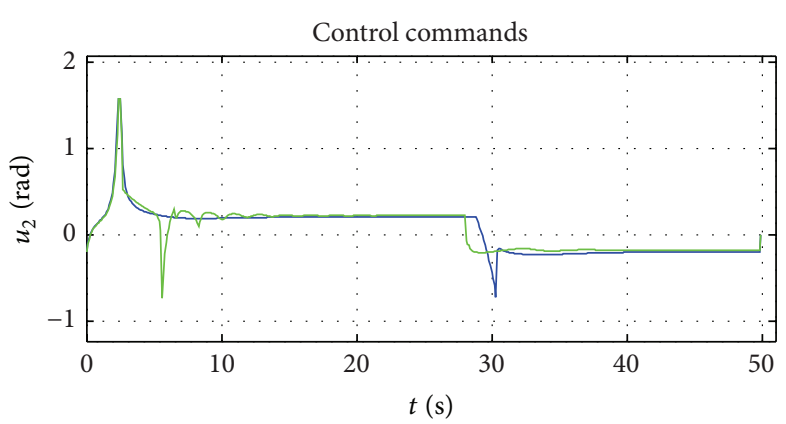

(A)

(B)

(c)

FIGURE 15: Trajectories in the $x-y$ plain and time responses of control commands by the PGR in (A) and the improved PGR with the adjustable look-ahead method in (B).

The first sudden change depicts that the trajectory switches from arc $o_{1}$ to $o_{2}$ and the second sudden change depicts that the trajectory changes from inside to outside of $o_{2}$. The steering angle of $u_{2}$ has only a sudden change in 28 seconds at intersection point $p$, which indicates that the improved PGR method with adjustable look-ahead method has good performance to converge the target $S$-shape passage. Similarly, the PGR and the improved PGR also can be applied in the complicate passage which consists of many arcs.

5.5. Discussion on Numerical Simulation Results. According to the above simulation results, we discuss the convergence property of the two methods for two-wheeled robot in this section.

The first issue concerns the influence of $\lambda$ in the two methods. By the PGR method in Section 3, the trajecotry is closer to the target circle if the value of $\lambda$ is larger, but the trajectory does not converge to the target circle/arc. However, by the improved PGR with adjustable look-ahead method, even if $\lambda$ is large or small, the robot trajectory is able to converge to the target circle/arc perfectly. The change of the value of $\lambda$ has no influence on the convergence property. The improved PGR with the adjustable look-ahead method for two-wheeled robot has better performance.

The second issue concerns the influence of time interval $d_{t}$. By the PGR method in Section 3, convergence property has some improvement as $d_{t}$ becomes short, but the trajectory is not able to converge to the target circle/arc. However, by the improved PGR with adjustable look-ahead method, when $d_{t}$ becomes long, the trajectory fluctuates around the target circle/arc. If we choose the proper $d_{t}$ value, the robot trajectory is able to converge to the target circle/arc perfectly.

The third issue concerns the capacity of resisting disturbance by the two methods. The two sizes of bound random disturbances are imposed; the results point out to two methods which have strong capacity of resisting disturbance. 
The fourth issue concerns the application in S-shaped passage. The simulations are performed on the PGR and the PGR with the adjustable look-ahead method. The results show that robot trajectory is consistent with the target Sshaped passage on the improved PGR with adjustable lookahead method.

In general, the improved PGR with adjustable look-ahead method has better performance along circle/arc passage. Compared with the other research, like references [12-19], the improved PGR with the adjustable look-ahead method has not only the merits of convergence perfectly, but also the strong capacity of resisting disturbance. However, this method has the limitation in application that is just for circle/ arc passage problem. It will be extended to the complicated passage environment in the future work.

\section{Conclusion and Future Work}

This paper presented the PGR and the improved PGR with the adjustable look-ahead method to track the circle/arc passage for two-wheeled robots. The robot's trajectory tended to deviate outward from the target circle in the first method. To improve this weakness, the adjustable coefficient angle $\beta$ is introduced. The robot adjusted the local coordinate system constantly according to the deviate between the trajectory and the target circle/arc, which made the trajectory converge to the target circle/arc perfectly. The simulation results also verified the improved PGR with adjustable look-ahead method had better performance on path tracking along the circle/arc passage. This approach was also validated on the Sshaped passage, which indicated that it could be applied to multiarc passage tracing problem.

In this paper, we focused on the control method to track the circle/arc passage and verify the efficiency on simulation environment without considering obstacles. However it does not mean that we neglect the obstacle avoidance problem. Actually, some members in our research group have proposed and are testing the obstacle avoidance algorithms with the PGR. In the future work, we will perform the experiments to verify the effectiveness of the method proposed in this paper in the real environment in the future.

\section{Conflict of Interests}

The authors declare that there is no conflict of interests regarding the publication of this paper.

\section{References}

[1] R. M. Bowen, J. Jollineau, and L. Margheim, "iRobot corporations intellectual property: accounting for research and development under U.S. GAAP versus IFRS," Journal of Business Case Studies, vol. 9, no. 4, pp. 321-331, 2013.

[2] Double Robotics, “Double," Mountain View, Calif, USA, 2014, http://www.doublerobotics.com/.

[3] J. B. Petelin, M. E. Nelson, and J. Goodman, "Deployment and early experience with remote-presence patient care in a community hospital," Surgical Endoscopy, vol. 21, no. 1, pp. 5356, 2007.
[4] X. V. Ha, C. Ha, and S. Choi, "Path tracking control of a mobile robot by using dual estimation algorithm," Advances in Mechanical Engineering, vol. 2013, Article ID 367127, 11 pages, 2013.

[5] A. Saudi and J. Sulaiman, "Robot path planning using four point-explicit group via nine-point laplacian (4EG9L) iterative method," Procedia Engineering, vol. 41, pp. 182-188, 2012.

[6] M. Sampei, "Feedback control of nonholonomic systems," Journal of the Society of Instrument and Control Engineers, vol. 39, no. 6, pp. 396-403, 1997.

[7] R. W. Brockett, "Asymptootic stability and feedback stabilization," in Differential Geometric Control Theory, pp. 181-191, Birkhäuser, Boston, Mass, USA, 1983.

[8] A. Takashima, Y. Hashimoto, K. Hori, N. Hanajima, H. Hikita, and M. Yamashita, "Feedback control of non-holonomic mobile robots by path-generating regulator," Transactions of the Japan Society of Mechanical Engineers C, vol. 70, no. 1, pp. 120-126, 2004 (Japanese).

[9] Y. Taruumi, N. Hanajima, J. Dai, and A. Takashima, "Driving control of car-like robots by Path-generating regulator," Transactions of the Japan Society of Mechanical Engineers C, vol. 79, no. 801, pp. 1693-1708, 2013 (Japanese).

[10] J. Dai, N. Hanajima, Y. Taruumi, and T. Kazama, “The improvement of Path-generating regulator for four-wheeled car-like robots by the EKF-SLAM," in Proceedings of the International Session of the Robotics Society of Japan (RSJ '12), pp. 103-106, September 2012.

[11] B. Yang, N. Hanajima, A. Yamamoto, M. Ayamura, and J. Dai, "Path-generating regulator along a straight passage for twowheeled mobile robots," in Proceedings of the 2013 IEEE IRS/RSJ International Conference on Intelligent Robots and Systems, pp. 4837-4844, 2013.

[12] J. P. Laumond, S. Sekhavat, and F. Lamiraux, Guidelines in Nonholonomic Motion Planning for Mobile Robots, vol. 229 of Lectures Notes in Control and Information Sciences, Springer, 1998.

[13] B. L. Ma, S. K. Tso, and W. L. Xu, "Adaptive/robust timevarying stabilization of second-order non-holonomic chained form with input uncertainties," International Journal of Robust and Nonlinear Control, vol. 12, no. 15, pp. 1299-1316, 2002.

[14] H. Khennouf and C. Canudes, "Quasi-continuous exponential stabilizers for nonholonomic systems," in Proceedings of the 13th IFAC World Congress, pp. 49-54, San Francisco, Calif, USA, 1996.

[15] J. A. Primbs, V. Nevistic, and J. C. Doyle, "Nonlinear optimal control: a control Lyapunov function and receding horizon perspective," Asian Journal of Control, vol. 1, no. 1, pp. 14-24, 1999.

[16] A. S. Matveev, M. Hoy, J. Katupitiya, and A. V. Savkin, "Nonlinear sliding mode control of an unmanned agricultural tractor in the presence of sliding and control saturation," Robotics and Autonomous Systems, vol. 61, no. 9, pp. 973-987, 2013.

[17] L. Ding, H. B. Gao, Z. Q. Deng, Z. J. Li, K. R. Xia, and G. R. Duan, "Path-following control of wheeled planetary exploration robots moving on deformable rough terrain," The Scientific World Journal, vol. 2014, Article ID 793526, 13 pages, 2014.

[18] J. Yang and J. Kim, "Sliding mode control for trajectory tracking of nonholonomic wheeled mobile robots," IEEE Transactions on Robotics and Automation, vol. 15, no. 3, pp. 578-587, 1999.

[19] H. Miao and Y. C. Tian, "Dynamic robot path planning using an enhanced simulated annealing approach," Applied Mathematics and Computation, vol. 222, pp. 420-437, 2013. 


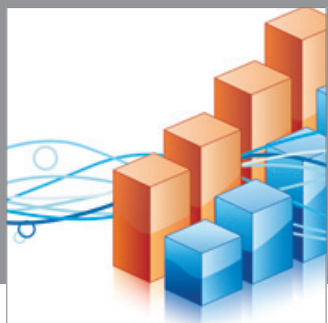

Advances in

Operations Research

mansans

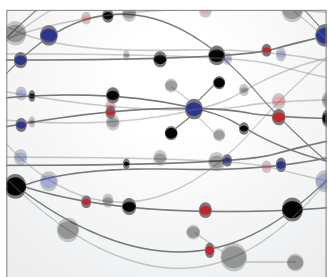

The Scientific World Journal
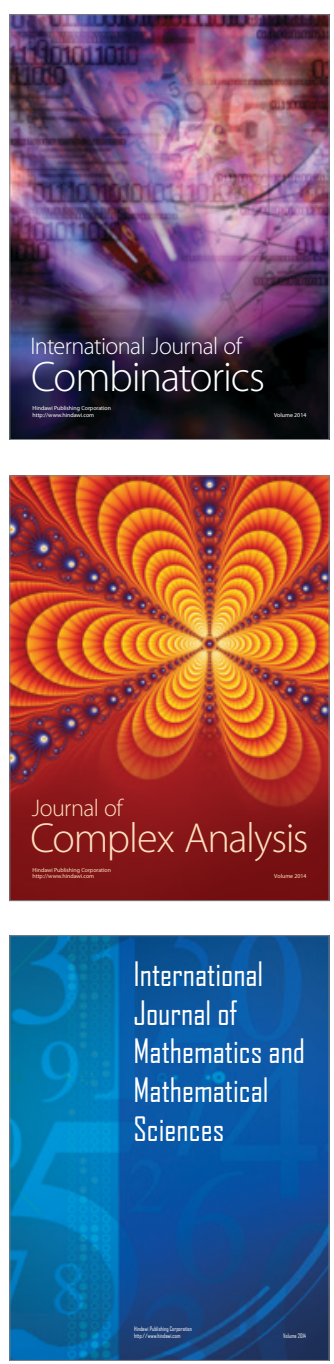
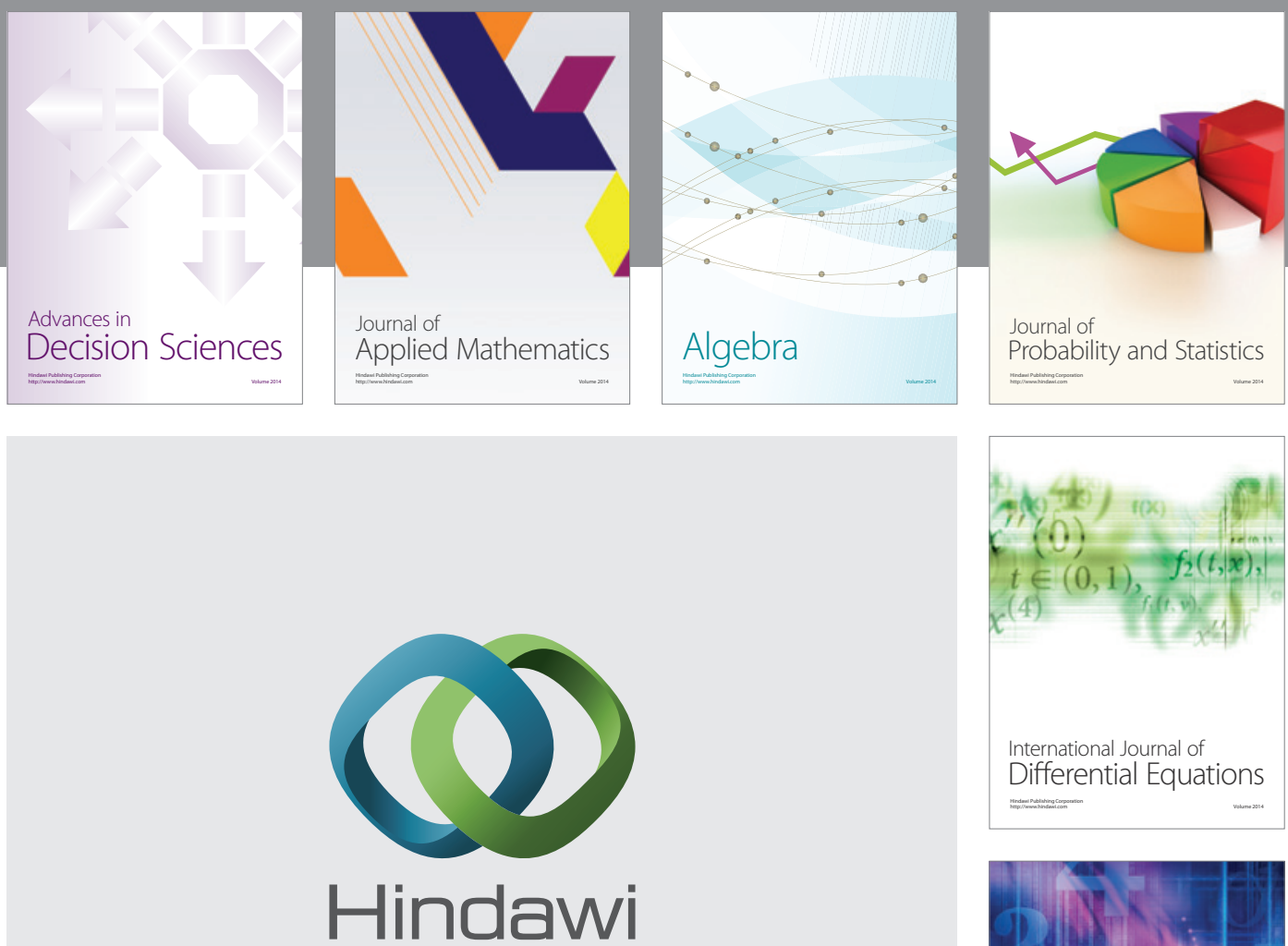

Submit your manuscripts at http://www.hindawi.com
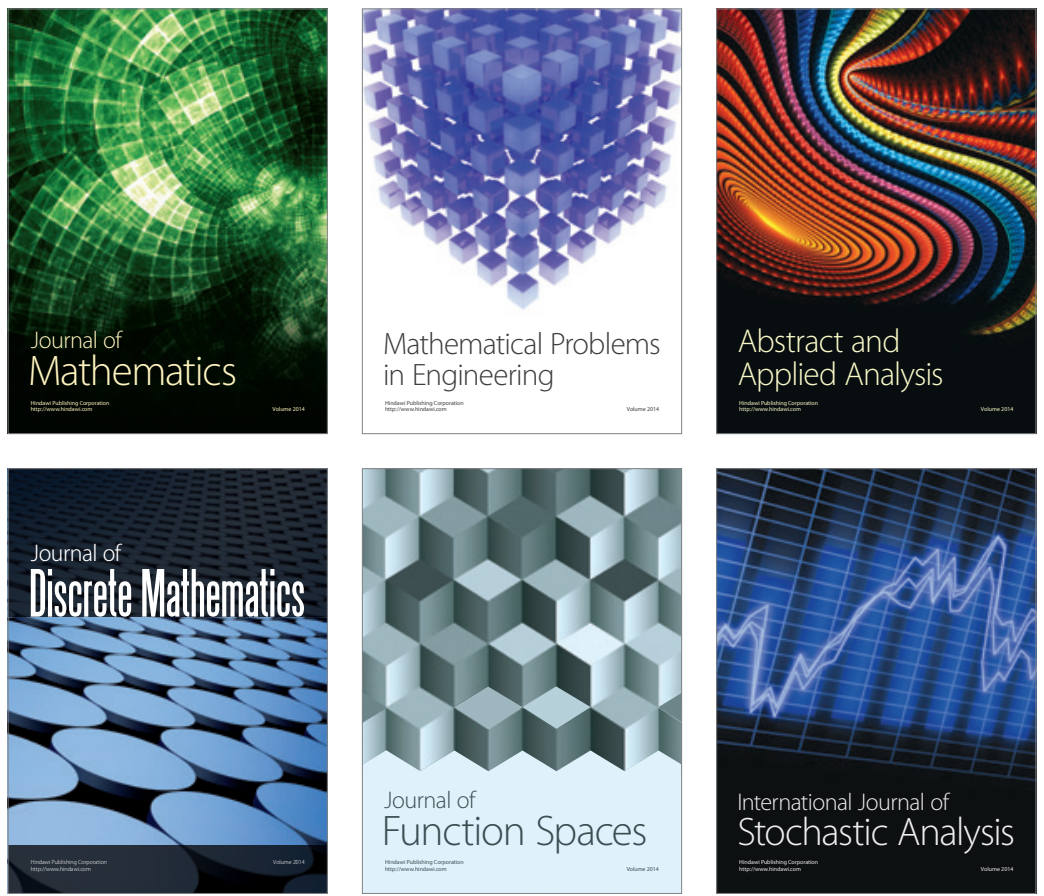

Journal of

Function Spaces

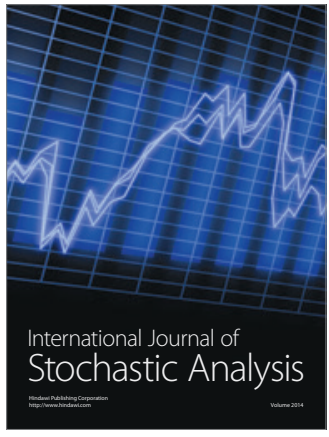

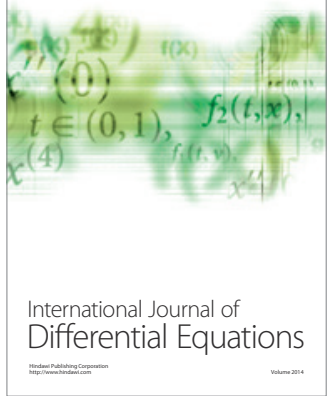
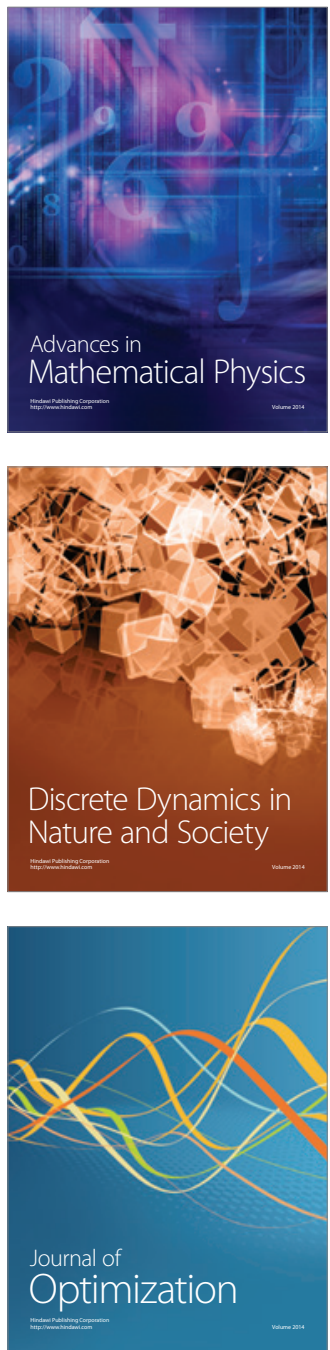